\title{
Neonatal seizures: the Dublin Collaborative Study
}

\author{
P D CURTIS, ${ }^{*}$. T G MATTHEWS,${ }^{*}$ T A CLARKE,${ }^{*}$ M DARLING,${ }^{*}$ P CROWLEY, $\dagger$ E GRIFFIN,$\dagger$

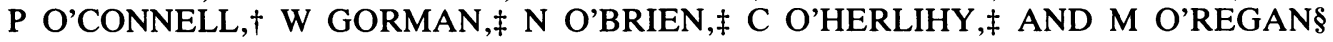

${ }^{*}$ Rotunda Hospital, †Coombe Lying-In Hospital, $¥$ National Maternity Hospital, and §Department of Statistics, Trinity College, Dublin

SUMMARY Asphyxial seizures occurred in 89 of 101829 infants born alive at term $(0 \cdot 87 / 1000)$ in three large maternity hospitals from January 1980 to December 1984 . These seizures were significantly associated with antenatal complications, primiparity, and prolonged pregnancy. Meconium staining of the amniotic fluid was also associated with asphyxial seizures, but there were high false positive $(11 \%)$ and false negative $(50 \%)$ rates. Fifteen of the infants who had seizures died $(18 \%)$ and $21(25 \%)$ were handicapped at 1 year. Outcome was most successfully predicted by the way the infant was feeding at 1-2 weeks. All infants taking more than half their estimated requirements by mouth at 1 week were normal, and those still being fed by tube at 2 weeks were handicapped.

Asphyxia is the commonest cause of brain injury in the perinatal period. Prevention, particularly in infants born at term, is the major goal of perinatal care. Perinatal asphyxia has been variously defined as including intrapartum events such as abruptio placentae, accidents with the cord, abnormal patterns of fetal heart rate, low fetal scalp $\mathrm{pH}$, and the passing of meconium by the fetus. Definitions have also included assessments of the infant's poor condition at birth such as the need for and the duration of resuscitation, the Apgar score, the acid base measurement, and varying combinations of abnormal neurological signs including seizures.

Many of these indices correlate poorly with both proven intrapartum asphyxia (hypoxia, acidosis, and ischaemia) and with brain damage among survivors. ${ }^{1-4}$ A seizure occurring within 48 hours of birth in an infant born at term is the most rigorously validated event that reflects asphyxia that can be prevented by better obstetric technique. Such seizures are also the most strongly predictive of either neonatal death or survival with handicap. ${ }^{56}$ In this study, which was carried out in three large maternity hospitals, we attempted to document the incidence and outcome of neonatal asphyxial seizures with a view to predicting and therefore preventing them. By identifying reliable prognostic factors in the immediate neonatal period, we hoped to counsel parents more accurately.

\section{Patients and methods}

The study population comprised all infants born alive at more than 37 completed weeks' gestation in the Coombe, the National, and the Rotunda maternity hospitals during the five years January 1980 to December 1984. Seizure was diagnosed when the consultant paediatrician found evidence of one of the following: myoclonic, multifocal clonic, generalised tonic, or focal clonic seizures. Infants with subtle seizures or 'jitteriness' were excluded. All infants were observed daily for at least four days postpartum, and those causing concern were seen daily by a consultant. Infants suspected of having seizures were admitted to the neonatal intensive care unit for assessment including estimations of blood glucose, sodium, and calcium concentrations; full blood count; lumbar puncture, neurological examination, and (latterly) cranial ultrasound examination. Infants of 37 weeks' gestation or more whose perinatal history suggested seizure due to intrapartum asphyxia and who had a fit within 48 hours of delivery were included in the study. All three hospitals collect data prospectively on infants with asphyxial seizures, which are presented at a monthly perinatal morbidity conference. The next normally formed infant of the same gestational age born after each infant who had a seizure was used as the control. Data about outcome of survivors was obtained up to a minium of one year from the records of the developmental follow up clinics at each hospital. A poor outcome among those who had seizures was defined as death, or handicap including cerebral palsy, mental retardation, epilepsy, or hearing or visual deficit. 
Antenatal variables documented in index cases and controls included maternal age, height, weight, weight gain in pregnancy, parity, incidence of cigarette smoking, and specific obstetric complications (hypertension, proteinuria, and antepartum haemorrhage). Intrapartum variables analysed included spontaneous or induced onset of labour, oxytocin stimulation, meconium stained amniotic fluid, fetal heart rate (monitored either by intermittent auscultation or by continuous electronic monitoring), mode of delivery, (normal, instrumental, or caesarean), and gestational age.

Neonatal variables recorded were sex, birth weight, Apgar score, need for intubation or resuscitation, and interval to the onset of the first seizure. Management of infants with seizures was similar in all three hospitals and included treatment with phenobarbitone $(10-20 \mathrm{mg} / \mathrm{kg})$, phenytoin $(10 \mathrm{mg} / \mathrm{kg})$ and diazepam $(0 \cdot 1 \mathrm{mg} / \mathrm{kg}) ;$ prophylactic anticonvulsant drugs were not given.

In order to assess the prognostic significance of methods of feeding, infants were assigned to one of three groups (assuming a requirement of $150 \mathrm{ml} / \mathrm{kg}$ / day of milk): those taking at least half their requirements orally by 1 week of age; those taking at least half their requirements orally by 2 weeks of age, and those who were still receiving more than half the daily requirements by nasogastric tube at 2 weeks of age. The $\chi^{2}$ test was used to estimate the significance of differences in proportions.

\section{Results}

During the study period a total of 101829 infants were born alive after more than 37 completed weeks' gestation; asphyxial seizures occurred within 48 hours of birth in $89(0 \cdot 87 / 1000)$. These were compared with 88 control babies. The antepartum variables that were significantly associated with the development of seizures are shown in table 1 . There was no association betweeen the development of seizures and either cigarette smoking or weight gain of less than $10 \mathrm{~kg}$ during pregnancy.

The intrapartum variables that were significantly associated with the development of seizures are shown in table 2. Meconium staining did not occur in half the babies with seizures though it was found in $11 \%$ of the controls and those who had seizures were more likely to be born by emergency caesarean section or with the assistance of forceps than control babies. There was no association between induction of labour and development of seizures.

The babies who had seizures weighed significantly less at birth than controls (table 2) but when the neonatal data of those who had seizures were analysed with regard to outcome (table 3 ), the association
Table 1 Comparison of antenatal details between the groups

\begin{tabular}{|c|c|c|c|}
\hline & $\begin{array}{l}\text { Babies with } \\
\text { seizures }(n=89)\end{array}$ & $\begin{array}{l}\text { Control } \\
\text { group }(n=88)\end{array}$ & p Value \\
\hline \multicolumn{4}{|l|}{ Maternal age: } \\
\hline$<20$ & 8 & 5 & NS \\
\hline $20-24$ & 21 & 34 & NS \\
\hline $25-29$ & 27 & 20 & NS \\
\hline $30-34$ & 16 & 22 & NS \\
\hline$\geqslant 35$ & 17 & 7 & $<0 \cdot 05$ \\
\hline \multicolumn{4}{|l|}{ Parity: } \\
\hline 0 & 49 & 36 & $<0.05$ \\
\hline $1-4$ & 27 & 40 & NS \\
\hline$>4$ & 13 & 12 & NS \\
\hline Complications & 34 & 15 & $<0.01$ \\
\hline No complications & 55 & 73 & NS \\
\hline
\end{tabular}

Table 2 Comparison of intrapartum details and birth weight between the groups

\begin{tabular}{|c|c|c|c|}
\hline & $\begin{array}{l}\text { Babies with } \\
\text { seizures }(n=89)\end{array}$ & $\begin{array}{l}\text { Control } \\
\text { group }(n=88)\end{array}$ & $p$ Value \\
\hline $\begin{array}{l}\text { Amniotic fluid } \\
\text { stained with } \\
\text { meconium }\end{array}$ & 44 & 10 & $<0.001$ \\
\hline $\begin{array}{l}\text { Emergency caesarean } \\
\text { section }\end{array}$ & 24 & 4 & $<0.001$ \\
\hline $\begin{array}{l}\text { Mid or high cavity } \\
\text { forceps delivery } \\
\text { Gestation longer than }\end{array}$ & 24 & 9 & $<0.001$ \\
\hline 41 weeks & 24 & 6 & $<0 \cdot 001$ \\
\hline Birth weight $(\mathrm{g})$ : & & & \\
\hline$<3000$ & 25 & 12 & $<0.05$ \\
\hline $3000-3500$ & 35 & 32 & NS \\
\hline$>3500$ & 29 & 44 & NS \\
\hline
\end{tabular}

Table 3 Association between measurements and outcome in 89 babies who had seizures

\begin{tabular}{lll}
\hline $\begin{array}{l}\text { Good } \\
\text { outcome }\end{array}$ & $\begin{array}{l}\text { Poor } \\
\text { outcome }\end{array}$ & p Value \\
\hline
\end{tabular}

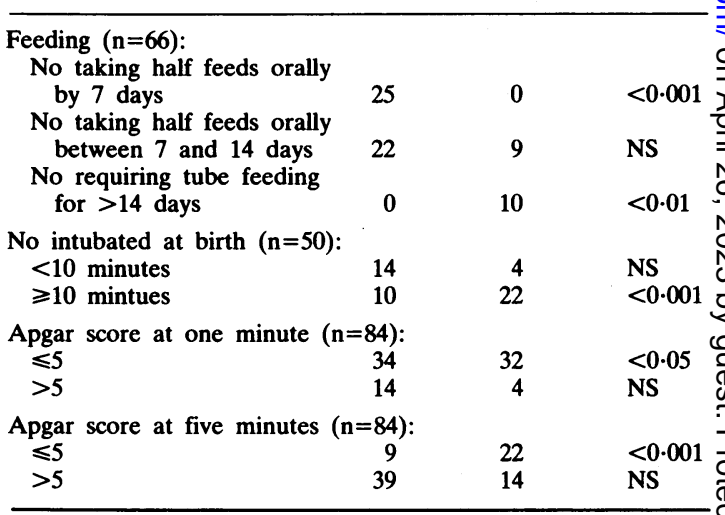


between low birth weight and poor outcome did not reach significance. Not surprisingly low Apgar scores at one minute $(p<0.05)$ and 5 minutes $(p<0.001)$ and the need for intubation for longer than 10 minutes at birth $(p<0.001)$, were significantly associated with a poor outcome, but the age of occurrence of seizures was not. In 66 infants the method of feeding at 1 and 2 weeks of age was documented; all infants who were satisfactorily feeding orally at 1 week were normal, and all those requiring tube feeding at day 14 were abnormal $(p<0.001)$.

Of the 89 infants who had seizures 15 died $(18 \%)$, 21 survived but with handicaps $(24 \%)$, and five were lost to follow up (all five were in the group who had rapidly returned to normal within the first week, four of whom have also been normal at six weeks, one did not attend). About a third of the surviving infants were handicapped.

There were significant differences among the rates of seizures among the three hospitals (table 4), as well as in the proportion of infants of 42 weeks' gestation or more in the seizure group (0 to $60 \%)$. This may reflect differences in the populations but must also reflect different management policies. The suggestion finds some support in the significant differences among the three hospitals in the number of caesarean sections performed $(p<0.001)$, the number of induced labours $(p<0.001)$, and the number of forceps deliveries $(p<0 \cdot 001)$. Among the infants born at more than 41 weeks' gestation who developed seizures $(n=24)$, seven $(29 \%)$ had a poor outcome compared with 29 of 65 infants born at term $(45 \%)$; this was not significant.

\section{Discussion}

Developments in perinatal medicine over the past decade have largely concerned the prevention of perinatal asphyxia and the more rigorous assessment of the association between preventable asphyxia and the incidence of long term brain damage-for example, cerebral palsy. ${ }^{34}$ Given that many of our assumptions regarding the causes of cerebral palsy, mental retardation, and epilepsy are based on outdated knowledge $^{1}$ and that there are many different definitions of perinatal asphyxia in current use, it is not surprising that confusion exists about the perinatal prevention of handicap. In addition, the introduction and widespread use of continuous fetal heart rate monitoring in labour has been justified by claims that it halves perinatal 'morbidity' despite the fact that we have no reliable estimate of how many complications originate in the perinatal period nor how many are really preventable.

Of the various definitions of intrapartum asphyxia currently used including abnormalities of the fetal heart rate, low fetal scalp blood $\mathrm{pH}$, and meconium staining of the amniotic fluid, not only are none strongly predictive of the condition of the baby at birth but their association with long term outcome is unknown. ${ }^{2} 389$ Of the various methods of defining 'poor condition at birth' (birth asphyxia), both a low Apgar score and the need for intubation (but not acid base measurement) have been shown to be only weakly predictive of long term handicap. ${ }^{10-14}$

The randomised controlled trial of intrapartum fetal heart rate monitoring carried out in Dublin was the first clinical study large enough to allow assessment of the validity of these various indices of perinatal asphyxia. This study strongly supported the hypothesis that neonatal seizures within 48 hours of delivery reflect intrapartum asphyxia. There were no significant differences between the monitored and unmonitored groups for abnormal neurological signs (other than seizures) in the neonatal period, low Apgar score, low $\mathrm{pH}$ in the umbilical vein, apnoea at birth, or endotracheal intubation. Dennis and Chalmers ${ }^{6}$ proposed that the early (within 48 hours) rate of seizures in infants born at term would provide a sensitive indicator of the quality of obstetric care, and the Dublin study of monitoring seems to support this. In addition, the fact that the incidence of seizures in the three hospitals in the present study were significantly different $(0.55$, 0.91 , and $1.2 / 1000$ babies born alive at term, respectively, $\mathrm{p}<0.05$ ) supports the hypothesis that asphyxial seizures are at least partially preventable.

The rate of seizures in the present study of $0 \cdot 87 / 1000$ babies born alive at term (43\% having a poor outcome) compares favourably with other similar studies. Levene et al reported a poor outcome $\left(1 / 1000\right.$ live births at $\left.\operatorname{term}^{14}\right)$ among

Table 4 Incidence of seizures and obstetric interventions in the three hospitals January 1980-December 1984

\begin{tabular}{lccrr}
\hline & Hospital A & Hospital B & Hospital C & $p$ Value \\
\hline Incidence of seizures/1000 live births & 0.55 & 0.91 & $1 \cdot 2$ & $<0.05$ \\
Percentage of induced labours & $22 \cdot 4$ & $10 \cdot 0$ & $9 \cdot 7$ & $<0.001$ \\
Percentage of forceps deliveries & $6 \cdot 8$ & $6 \cdot 3$ & $16 \cdot 1$ & $<0.001$ \\
Percentage of caesarean sections & $7 \cdot 8$ & $5 \cdot 2$ & 8.6 & $<0.001$ \\
\hline
\end{tabular}

The $\mathrm{p}$ values refer to significant differences in group rates for each variable. 
asphyxiated infants delivered in Leicester from 1980 to 1984, and rates of 'moderate' and 'severe' postasphyxial encephalopathy of $1 \cdot 15$ and $1 \cdot 0 / 1000$ babies born alive at term, respectively. All the former and many of the latter group would be expected to have neonatal seizures though some would be 'subtle seizures'. Leveno et al ${ }^{15}$ reported a rate of seizures of $1 / 1000$ live births weighing more than $2500 \mathrm{~g}$, and used this 'low' rate as one of the main justifications of a rate of caesarean section of $18 \%$. The present study included five infants born at term weighing less than $2500 \mathrm{~g}$, and excluded a similar number of infants who had seizures but weighed more than $2500 \mathrm{~g}$ and were born prematurely. MacDonald $e t$ at $t^{5}$ showed that $93 \%$ of infants of more than 28 weeks' gestation who develop asphyxial seizures do so within $\mathbf{4 8}$ hours of delivery. That one hospital in this study had a rate of seizures of $0.55 / 1000$ babies born alive at term with a rate of caesarean section of $8 \%$ means that prevention of intrapartum asphyxia does not depend on the number of caesarean sections. This hospital had a high rate of induction of labour $(22 \%)$ directed at prevention of prolonged pregnancy, and used continuous monitoring of the fetal heart rate in $33 \%$ of labours in pregnancies at term. This raises the question of whether continuous monitoring of the fetal heart rate prevents asphyxial damage. In the trial of intrapartum monitoring a significant reduction in asphyxial seizures occurred in the group that was monitored, though this effect was confined to the $15 \%$ of labours that lasted more than five hours. Length of labour was not included in the present study as it was felt that the retrospective assessment of onset and duration of labour was insufficiently accurate.

In infants born at term asphyxial seizures have been shown to reflect preventable obstetrical intrapartum asphyxia in a prospective randomised trial. ${ }^{5}$ The present study confirms the association between asphyxial seizures and poor outcome (43\%) and we are now able to counsel parents on the likelihood of handicap using the infant's neurological state and the ability to feed normally as guides.

Asphyxial seizures should be identified as well as asphyxial deaths (before and during labour, and in the neonatal period) in all maternity units because they are a sensitive indicator of the value, or $\frac{\rho}{\overline{\frac{\partial}{0}}}$ otherwise, of perinatal intervention.

We thank the Masters of the three hospitals, Dr J Drumm, Dr G Henry, and Dr J Stronge for their help, and Miss Audrey Dixon for typing the manuscript.

\section{References}

1 Freeman JM. Prenatal and perinatal factors associated with brain disorders. Bethesda: National Institutes of Health, 1985.

2 Hey E. Foetal hypoxia and subsequent handicap: the problem of establishing a causal link. In: Chamberlain GVP, Orr CJB, $\vec{\omega}$ Sharp F, eds. Litigation and obstetrics and gynaecology. Pro- $\mathcal{D}$ ceedings of the fourteenth study group. London: Royal College ڤั of Obstetricians and Gynaecologists, 1985: 233-42.

${ }^{3}$ Niswander K, Henson G, Elbourne D, et al. Adverse outcome of pregnancy and the quality of obstetric care. Lancet $\omega$ 1984;ii:827-31.

${ }^{4}$ Paneth N, Stark RI. Cerebral palsy and mental retardation in $\vec{\circ}$ relation to indicators of perinatal asphyxia. Am J Obstet

Gynecol 1983;147:960-6.
MacDonald D, Grant A, Sheridan-Pereira M, Boylan P, 은 Chalmers I. The Dublin randomized controlled trial of intrapar- tum foetal heart rate monitoring. Am J Obstet Gynecol $\mathcal{C D}$ 1985;152:524-39.

6 Dennis J, Chalmers I. Very early neonatal seizure rate: a $\frac{\bar{D}}{\mathbb{D}}$ possible epidemiological indicator of the quality of perinatal 3 care. Br J Obstet Gynaecol 1982;89:418-26.

7 Quilligan EJ, Paul RH. Foetal monitoring: is it worth it? Obstet Gynecol 1975;45:96-100.

${ }^{8}$ Taylor DJ, Howie PW, Davidson J, Davidson D, Drillien CM. Do pregnancy complications contribute to neurodevelopmental disability? Lancet 1985;i:713-6.

9 Dennis J. The long-term effects of intrapartum cerebral damage. In: Crawford JW, ed. Risks of labour. Chichester: John Wiley and Sons, 1985:157-88.

${ }^{10}$ Nelson KB, Ellenberg JH. Apgar scores as predictors of chronic neurologic disability. Pediatrics 1981;6:31-44.

11 Lumley J, Wood C. Foetal acidosis. Aust NZ J Obstet Gynaecol 1969;9:145-51.

12 Brann AW. Factors during neonatal life that influence brain $\frac{0}{3}$ disorders. In: Freeman JM, ed. Prenatal and perinatal factors $\bar{\supset}$ associated with brain disorders. Bethesda: National Institutes of $\frac{F}{0}$ Health, 1985:263-358.

13 Sykes GS, Molloy PM, Johnson P, et al. Do Apgar scores indicate asphyxia? Lancet $1982 ;$;:494-6.

14 Levene MI, Sands C, Grindulis H, Moore JR. Comparison of $\frac{?}{\delta}$ two methods of predicting outcome in perinatal asphyxia. Lancet 1986;ii:67-9.

15 Leveno KJ, Cunningham FG, Pritchard JA. Caesarean section: an answer to the house of Horne. Am J Obstet Gynecol 1985;153:838-44.

Correspondence to Dr T G Matthews, Department of Paediatrics, Rotunda Hospital, Dublin I.

Accepted 31 March 1988. 